\title{
Medicinal prospective of Citrus limon and Citrus sinensis peels essential oil by Gas Chromatography/ Mass Spectrometry (GC/MS) compositional analysis
}

\author{
Uraku, A. J. ${ }^{\star}$, Uraku, O. H. ${ }^{1}$, Nwankwo, V. O. U. ${ }^{2}$, Okoye, C. J. ${ }^{3}$, Ozioma, P. E. ${ }^{4}$, \\ Edenta, C. ${ }^{5}$ and Ezeali, C. ${ }^{1}$
}

\author{
${ }^{1}$ Department of Biochemistry, Ebonyi State University, PMB 053 Abakaliki, Ebonyi State, Nigeria. \\ 2Department of Biotechnology, Ebonyi State University, PMB 053 Abakaliki, Ebonyi State, Nigeria. \\ ${ }^{3}$ Department of Biochemistry, University of Calabar, Calabar, Cross River State, Nigeria \\ ${ }^{4}$ Department of Biochemistry, Federal University, Wukari, Taraba State, Nigeria. \\ ${ }^{5}$ Department of Biochemistry, Renaissance University, Ugbawka, Enugu State, Nigeria.
}

\begin{abstract}
*Corresponding author. Email: urakuaj@yahoo.com; Tel: +234-8068073037; LiveDNA*: 234.25095; ORCID ID: 0000-0002-6518-4627.
Copyright @ 2020 Uraku et al. This article remains permanently open access under the terms of the Creative Commons Attribution License 4.0, which permits unrestricted use, distribution, and reproduction in any medium, provided the original work is properly cited.
\end{abstract}

Received 19th June, 2020; Accepted 27th July, 2020

\begin{abstract}
Citrus fruits are highly nutritious and medicinal fruits that are commonly cultivated throughout the tropics. This study was carried out to determine the bioactive compounds of the methanol peel extracts of Citrus limon and Citrus sinensis. The peels from fresh fruits of $C$. limon and $C$. sinensis were air-dried at room temperature and were pulverized into fine powder. The bioactive determinations were carried out by Gas Chromatography/Mass Spectrometry (GC/MS). The mass spectrum of the compounds found in the peels extract was matched with the National Institute of Standards and Technology (NIST) library. The results showed that four (4) and five (5) bioactive compounds were identified in $C$. limon and $C$. sinensis methanol peel extracts respectively. The $C$. limon peel extract contained 4-(methylsulfanyl) but-1en-3-yne, Sulfanylphenylium, 1,3-thiazole and 3-methylidenecyclobutanecarbonitrile while $C$. sinensis contained Pyridine2-carbaldehyde, 1-Methyl-1 H-pyrrole-2-carbaldehyde, Glutamic acid, 2-Ethyl-5-methyl-1 H-pyrrole and Pyrrolidin-2-one. The results of this study therefore unveiled that the $C$. limon and $C$. sinensis peel extracts contained variable bioactive compounds with medicinal value and could be useful raw materials for pharmaceutical industries.
\end{abstract}

Keywords: Citrus peels, methanol extract, medicinal phytochemicals, environmental wastes.

\section{INTRODUCTION}

The postulation that, for all need in all continents, there is a plant, is not only remarkably a hyperbolical statement but appears to be in affirmation in all ramifications. For millennia, people have been treated with herbal or animalderived medicines, via knowledge handed down through generations. Tamil et al. (2017) reported that about $80 \%$ of people living in developing countries rely on medicinal plants for treatment of various kinds of diseases currently because they are relatively cheap and effective. So, studies in the field of phytochemistry to find out the bioactive substances from medicinal plants have considerably increased. Presently, scores of modern methods are accessible for standardization of crude drugs. Gas Chromatography Mass Spectrum (GC/MS) has become firmly established to identify the active principles in natural products (Rohloff, 2015).

Citrus fruits are highly nutritious and medicinal fruit that are commonly cultivated throughout the tropics. It belongs to the genus Citrus, subgenus Papeda. Citrus fruits include orange, lemon, lime and grapefruits with tangerine and pomelo inclusive. Citrus fruits are mainly used by processing industries while the peels and pulps are 
generally discarded as waste products (Uraku et al., 2018). The fruits constitute only $0.9 \%$ of total daily calories and $1.7 \%$ of daily carbohydrate intake while the peel wastes are highly perishable and seasonal. These peel wastes could be wealth for the farmers if the processing industries and monitoring agencies develop tactics to use them and create awareness in bringing the useful products from waste materials (Suja et al., 2017). The wastes of citrus fruits are more than the juice yield in the fruit weight. Thus, a very large amount of the lemon and orange wastes are formed yearly (Arora and Kaur, 2013, Gotmare and Gade, 2018). Due to high wastes from citrus fruits in the environment, there is often an attention in bringing them into useful products and lemon and orange wastes are not exempted. To that effect, apt method needs to be adopted in the conversion of citrus peels and pulps into valueadded products (Gotmare and Gade, 2018). By so doing, the environmental pollution can be reduced. Citrus peels have been reported by Uraku and Igwenyi (2016) to be rich in nutrients and phytochemicals. Obviously, they can be efficiently used not only as drugs but also as food supplements. The citrus peels have been acclaimed by rural dwellers to serve as mosquito repellent and anticoagulant among others.

Previous study by Choike et al. (2017) on ethanol extraction and GC-MS analysis of $C$. sinensis peel oil reported fifteen compounds. Also, Kamaliroosta et al. (2016) identified seven compounds in the orange peel essential oil and eighteen compounds in sweet lemon peel. More so, Larijani (2004) appraised the essential oils of orange and sour lemon. The results revealed fourteen, twenty-two and twenty-one compounds in the $C$. sinensis (Thomson), C. sinensis (Valencia) and C. limon peel essential oil respectively. Mirza and Bahernik (2006) also assessed the chemical composition of the essential oil of orange peel by GC/MS and identified twenty-one compounds. The essential oils of three varieties of $C$. sinensis in Kenya (washington, valencia and salutiana navel) were evaluated by GC/MS and 56, 72 and 73 compounds were identified in salutianat, valencia and washington respectively (Njoroge et al., 2005).

To the best of our knowledge, of all the studies done on $\mathrm{GC} / \mathrm{MS}$ analysis of these peels, no research has been done on GC/MS analysis of $C$. limon and $C$. sinensis peels from Nigeria. Thus, this research was designed to determine the phytochemicals or bioactive components of $C$. limon and $C$. sinensis peels. The bioactive compounds may be postulated to be responsible for some of the ethnomedicinal potentials of citrus peels.

\section{MATERIALS AND METHODS}

\section{Collection and identification of plant material}

Fresh fruits of Citrus limon and Citrus sinensis were purchased at Abakpa Main Market in Abakiliki, Ebonyi State, Nigeria. The plant samples were identified and authenticated by Taxonomist, Dr. (Mrs) C. V. Nnamami at the Department of Biological Sciences, Ebonyi State University, Abakaliki, Ebonyi State, Nigeria.

\section{Preparation of plant extract}

The peels of $C$. limon and $C$. sinensis fruits were obtained by using a sharp knife and washed thoroughly with distilled water to remove dirt and debris. The peels were sliced into smaller pieces before they were air-dried for two weeks at room temperature $\left(28 \pm 3^{\circ} \mathrm{C}\right)$. The dried peels were pulverized into fine powder using electric blenders (CORONA-REF. 121, Landers and Qlink blender, Model No. OBL-15L40). The powdered materials were stored in air tight polyethene bags protected from direct sunlight until required for use.

Five (5) grams of the powdered peels were extracted with $100 \mathrm{ml}$ of $98 \%$ methanol overnight in a stopped bottle and with occasional stirring at room temperature $\left(28 \pm 3^{\circ} \mathrm{C}\right)$. The samples were first sieved using muslin cloth and then filtered using Whatman No.1 filter paper. This process was repeated three times to ensure that the pulverized peels are properly extracted. The filtrates were concentrated under reduced pressure at $40^{\circ} \mathrm{C}$ for 45 minutes in a rotary vacuum evaporator and then lyophilized to get a brown solid extract. The yield of the extracts was expressed in terms of the percentage of the dry weight of initial plant materialused. The dry extracts obtained were kept in a refrigerator at $4^{\circ} \mathrm{C}$ until required for use.

\section{Gas chromatography/Mass spectrometry (GC/MS)}

GC/MS analysis was carried out on a GC-MS (Model: QP2010 PLUS Shimadzu, Japan) comprising a AOC-20i auto-sampler and chromatograph interfaced to a mass spectrometer (GC/MS). The instrument was equipped with a VF $5 \mathrm{~ms}$ fused silica capillary column of $30 \mathrm{~m}$ length, $0.25 \mathrm{~mm}$ diameter and $0.25 \mu \mathrm{m}$ film thickness. The temperatures employed were column oven temperature $80^{\circ} \mathrm{C}$ and injection temperature $250^{\circ} \mathrm{C}$ at a pressure of $108.0 \mathrm{kPa}$ with total flow and column flow of $6.20 \mathrm{ml} / \mathrm{min}$ and $1.58 \mathrm{ml} / \mathrm{min}$ respectively. The linear velocity was 46.3 $\mathrm{cm} / \mathrm{sec}$ and a purge flow of $3.0 \mathrm{ml} / \mathrm{min}$. The GC program ion source and interface temperature were $200.00^{\circ} \mathrm{C}$ and $250.00^{\circ} \mathrm{C}$ respectively with solvent cut time of $2.50 \mathrm{~min}$. The MS program starting time was $3.00 \mathrm{~min}$ which ended at $30.00 \mathrm{~min}$. with event time of $0.50 \mathrm{sec}$, scan speed of $1666 \mu \mathrm{l} / \mathrm{sec}$, scan range 40-800u and an injection volume of $1 \mu \mathrm{l}$ of the plant extract (split ratio 10:1). The total running time of $\mathrm{GC} / \mathrm{MS}$ was $30 \mathrm{~min}$. The relative percentage of the extract was expressed as percentage with peak area normalization.

\section{Identification of phytocompounds}

Interpretation on the mass spectrum was conducted using the database of National Institute Standard and 
Intensity

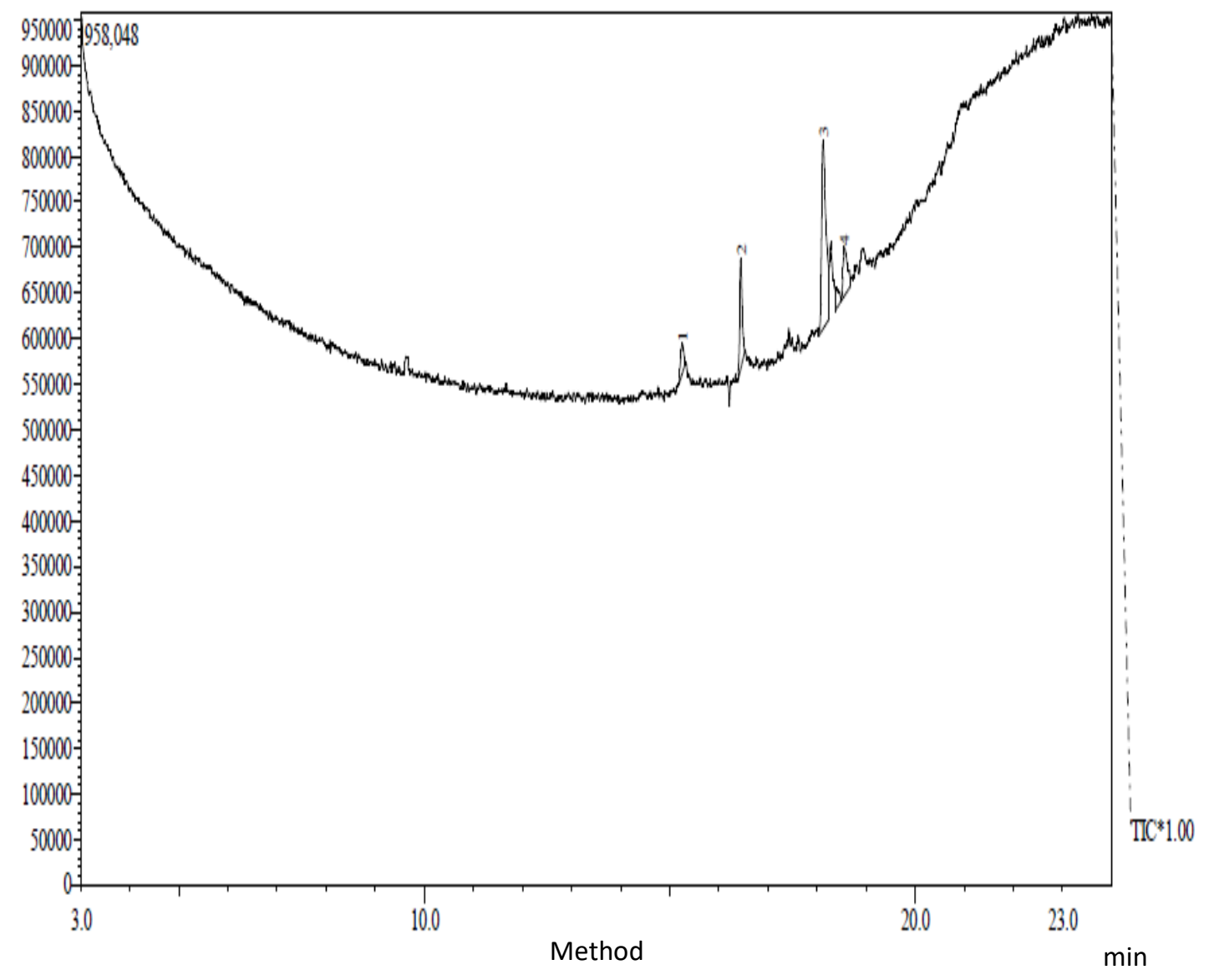

Figure 1. Chromatogram of methanol peel extract of $C$. limon essential oil.

Technology (NIST) having more than 62,000 patterns. The fragmentation pattern spectra of the unknown components were compared with those of known components stored in the NIST library (NIST Ver. 3.2 of 2010). The compound bioactivity prediction was based on Duke's Phytochemical and Ethnobotanical Databases (2014). The relative percentage amount of each phytocomponent was calculated by comparing its average peak area to the totalarea. The name, molecular weight and structure of the components of the test materials were ascertained.

\section{RESULTS}

\section{Chromatogram of methanol extract of essential oil peel}

The chromatogram of essential oil of Citrus limon and Citrus sinensis peel reveled four and five peaks respectively (Figures 1 and 2).

\section{Identified compounds}

The identified compounds in methanol peel extracts of $C$. limon and $C$. sinensis essential oil with retention time, peak area (\%), molecular weight and formular are depicted in Tables 1 and 2.

\section{Phytochemicals in methanol extract of $C$. limon and $C$. sinensis peel}

The proposed phytochemicals in methanol peel extracts of $C$. limon and $C$. sinensis with their name of compound, molecular structure and bioactivity are shown in Tables 3 and 4.

\section{DISCUSSION}

The gas chromatogram and mass spectra of methanol peel extracts of Citrus limon and Citrus sinensis were presented in Figures 1 and 2. Thus, four and five peaks were depicted respectively and these suggested presence of four and five compounds in the methanol peel extracts of $C$. limon and $C$. sinensis essential oil. The projected compounds, retention time (RT), peak area percentage, 


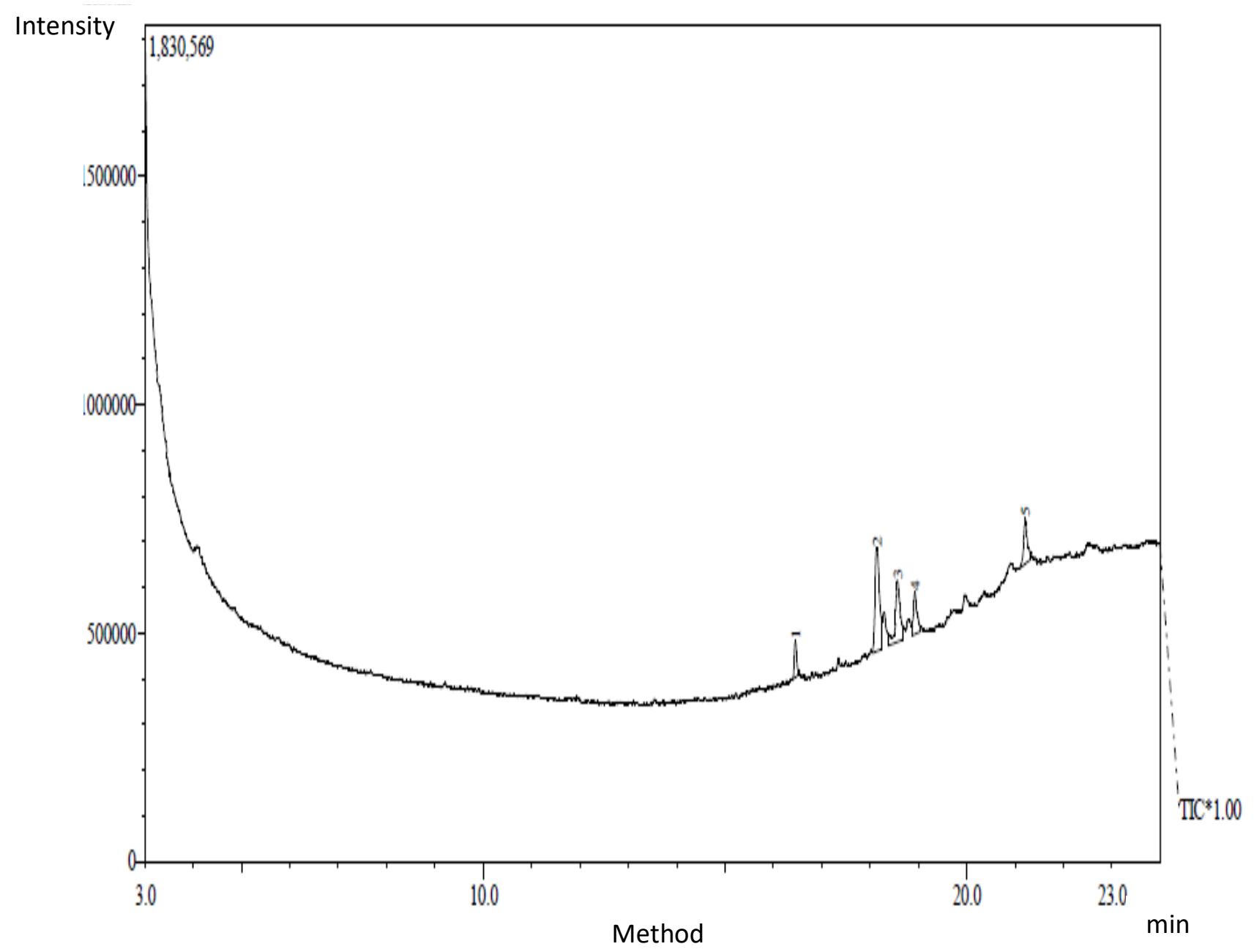

Figure 2. Chromatogram of methanol peel extract of $C$. sinensis essential oil.

Table 1. Identified compounds in methanol peel extract of $C$. limon essential oil with retention time, peak area (\%), molecular weight and formular by GC/MS analysis.

\begin{tabular}{llcccc}
\hline Peak No & Name of Compound & Retention time & Peak area \% & Molecular weight & Molecular formula \\
\hline 1 & 3-methylidenecyclobutanecarbonitrile & 15.258 & 6.48 & 93.12 & $\mathrm{C}_{6} \mathrm{H}_{7} \mathrm{~N}$ \\
2 & 1,3-thiazole & 16.447 & 18.13 & 85.12 & $\mathrm{C}_{3} \mathrm{H}_{3} \mathrm{NS}$ \\
3 & 4-(methylsulfanyl) but-1-en-3-yne & 18.126 & 56.53 & 98.16 & $\mathrm{C}_{5} \mathrm{H}_{6} \mathrm{~S}$ \\
4 & Sulfanylphenylium & 18.561 & 18.86 & 109.16 & $\mathrm{C}_{6} \mathrm{H}_{5} \mathrm{~S}$ \\
\hline
\end{tabular}

Table 2. Identified Compounds in methanol peel extract of $C$. sinensis essential oil with retention time, peak area (\%), molecular weight and formular by GC/MS analysis.

\begin{tabular}{llcccc}
\hline Peak No & Name of Compound & Retention time & Peak area \% & Molecular weight & Molecular formula \\
\hline 1 & Pyrrolidin-2-one & 16.454 & 7.46 & 85.10 & $\mathrm{C}_{4} \mathrm{H}_{7} \mathrm{NO}$ \\
2 & Pyridine-2-carbaldehyde & 18.144 & 40.16 & 107.11 & $\mathrm{C}_{6} \mathrm{H}_{5} \mathrm{NO}$ \\
3 & 1-Methyl-1 $\mathrm{H}$-pyrrole-2-carbaldehyde & 18.566 & 25.00 & 109.12 & $\mathrm{C}_{6} \mathrm{H}_{7} \mathrm{NO}$ \\
4 & 2-Ethyl-5-methyl-1 $\mathrm{H}$-pyrrole & 18.929 & 12.75 & 109.16 & $\mathrm{C}_{7} \mathrm{H}_{11} \mathrm{~N}$ \\
5 & Glutamic acid & 21.203 & 14.63 & 147.12 & $\mathrm{C}_{5} \mathrm{H}_{9} \mathrm{NO}_{4}$ \\
\hline
\end{tabular}


Table 3. Proposed phytochemicals in methanol peel extract of $C$. limon essential oil with their Name of compound, Molecular structure and Bioactivity.

Peak No. Name of compound

Nf: Not found. Source: Duke.s Phytochemical and Ethnobotanical Database (2014).

Table 4. Proposed phytochemicals in methanol peel extract of $C$. sinensis with their Name of compound, molecular structure and Bioactivity.

$\begin{array}{lll}\text { S/No Name of Compound } & \text { Bolecular structure } & \text { Bioactivity } \\ \begin{array}{l}\text { Pyridine-2- } \\ \text { carbaldehyde }\end{array} \text { Pyrrolidin-2-one } \\ \begin{array}{l}\text { 1-Methyl-1 H-pyrrole-2- } \\ \text { carbaldehyde }\end{array}\end{array}$


Table 4. Contd.

4 2-Ethyl-5-methyl-1Hpyrrole
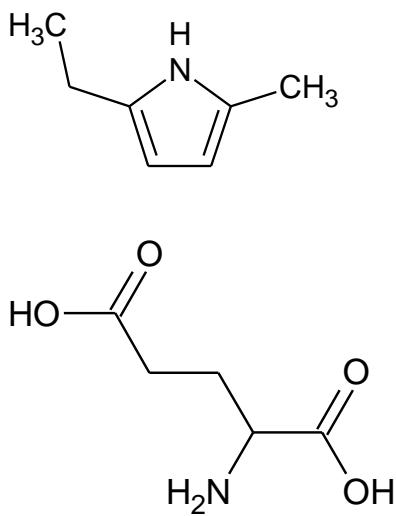

Hypoxemic, improve Cerebral Hypoxia, Increase T-helper, Increase Tyrosine Hyroxylase Activity, Suppress HMG-CoA Reductase Activity, Testosterone-Hydroxylase-Inducer, Tyrosine-Hyroxylase-Activator

Urine-Acidifier, Urinary-Acidulant, Inhibit Production of Uric Acid, Increase Aromatic Amino Acid Decarboxylase Activity, Arachidonic-Acid-Inhibitor, Arachidonic acidInhibitor

Nf: Not found. Source: Duke.s Phytochemical and Ethnobotanical Database (2014).

molecular weight and molecular formula were chatted in Table 1 and 2. The phytochemicals present in the methanol peel extracts of $C$. limon with peak area percentage were 4-(methylsulfanyl) but-1-en-3-yne (56.53), Sulfanylphenylium (18.86), 1,3-thiazole (18.13) and 3-methylidenecyclobutanecarbonitrile (6.48) while that of $C$. sinensis were Pyridine-2-carbaldehyde, 1Methyl-1H-pyrrole-2-carbaldehyde, Glutamic acid, 2Ethyl-5-methyl-1H-pyrrole and Pyrrolidin-2-one with peak area percentages of $40.16,25.00,14.63,12.75$ and 7.46 respectively.

Tables 3 and 4 showed the nature, structures and biological activities of the phytochemicals found in the methanol peel extracts of $C$. limon and $C$. sinensis essential oil respectively. Out of four compounds found in C. limon, only 4-(methylsulfanyl) but-1-en-3-yne with peak area percentage of 56.53 had an identify biological activities (Table 3 ) while in $C$. sinensis, five compounds were recognized and only three compounds had eminent bioactive roles (Table 4). However, there is no information on biological activity of other compounds in methanol peel extracts of $C$. limon and $C$. sinensis essential oil.

The outcome of this study is incongruity with report of Kumar and Vijayalakshmi (2011) on GC/MS analysis of ethanol extract of $V$. vinifera seed. Also, the report of Choike et al. (2017) is not in consonance with the results obtained in this study. Studies carried out by Qiao et al. (2008), Das et al. (2014), Prasad et al. (2016), Rashmi et al. (2017), Kadhim et al. (2017), Uraku et al. (In press) are in divergence with the outcome of this study. The detergency of this study with the reports of other researchers is in both number and natures of phytoconstituents found. The discrepancy in these peel extracts could be due to differences in plant parts, solvents of extraction and geographical location.

\section{Conclusion}

The bioactive compounds identified by GC/MS analysis of the peels have demonstrated that peels are important raw materials in drug formation. This study unveiled that the essential oil of $C$. limon and $C$. sinensis peels contained phytchemicals that could be beneficial to man. This study will help researchers to covert waste to wealth.

\section{CONFLICT OF INTEREST}

No conflict of interest associated with this work.

\section{REFERENCES}

Arora, M., \& Kaur, P. (2013). Phytochemical screening of orange peel and pulp. International Journal of Research in Engineering and Technology, 2(12), 517-522.

Choike, P. B., Bhor, A. K., Shete, A. M., Sonawane, R. K. (2017). Extraction and GC-MS analysis of orange (Citrus sinensis) peel oil. Research Journal of Life Sciences, Bioinformatics, Pharmaceutical and Chemical Science, 2(5), 41-51.

Das, D. R., Sachan, A. K., Shuaib, M., \& Imtiyaz, M. (2014). Chemical charecterization of volatile oil components of Citrus reticulata by GC-MS analysis. World Journal of Pharmacy and Pharmaceutical Sciences, 3(6), 1197-1204.

Duke's Phytochemical and Ethnobotanical Database (2014). Retrieved from https://data.nal.usda.gov/dataset/dr-dukesphytochemical-and-ethnobotanical-databases.

Gotmare, S., \& Gade, J. (2018). Orange peel: A potential source of phytochemical compounds. International Journal of ChemTech Research, 11(02), 240-243

Kadhim, M. J., Al-Rubaye, A. F., \& Hameed, I. H. (2017). Determination of Bioactive Compounds of Methanolic Extract of Vitis Vinifera Using GC-MS. International Journal of Toxicological and Pharmacological Research, 9(2), 113-126.

Kamaliroosta, L., Zolfaghari, M., Shafiee, S., Larijani, K., \& Zojaji, M. (2016). Chemical Identifications of Citrus Peels Essential Oils. Journal of Food Biosciences and Technology, 6(2), 6976.

Kumar, K. A., \& Vijayalakshmi, K. (2011). GC-MS analysis of phytochemical constituents in ethanolic extract of Punica granatum peel and Vitis vinifera seeds. International Journal of Pharma and Bio Sciences, 2(4), 461-468.

Larijani, K. (2004). Extraction, purification and identification of natural compounds, as wellas analysis of the essential oils of 
some Iran's native plants. Ph.D Thesis of Science and Research Branch, Islamic Azad University, Tehran, Iran.

Mirza, M., \& Bahernik, Z. (2006). The role of removing terepene in chemical composition of citrus peel essential oil. Iran's Medicinal and Aromatic Plants Research, 22(3), 225-250.

NIST (2010). Automated mass spectral library with search program (Data version: NIST11, version 2.0). National Institute of Standards and Technology, Gaithersburg, MD., USA.

Njoroge, S. M., Koaze, H., Mwaniki, M., Minh Tu, N. T., \& Sawamura, M. (2005). Essential oils of Kenyan Citrus fruits: volatile components of two varieties of mandarins (Citrus reticulata) and a tangelo (C. paradisix C. tangerina). Flavour and Fragrance Journal, 20(1), 74-79.

Prasad, D. A., Prasad, B. R., Prasad, D. K., Shetty, P., \& Kumar, K. S. (2016). GC-MS compositional analysis of essential oil of leaf and fruit rind of Citrus maxima (Burm.) Merr. from Coastal Karnataka, India. Journal of Applied Pharmaceutical Science, 6(5), 68-72.

Qiao, Y., Xie, B. J., Zhang, Y., Zhang, Y., Fan, G., Yao, X. L., \& Pan, S. Y. (2008). Characterization of aroma active compounds in fruit juice and peel oil of Jinchen sweet orange fruit (Citrus sinensis (L.) Osbeck) by GC-MS and GCO. Molecules, 13(6), 1333-1344.

Rashmi, Y., Reena, S. L. \& Muhammad S. D. (2017). GC/MS analysis, antioxidant and antibacterial activities of essential oil extracted from Citrus paradisiimacfad fruit peels. International Journal of Scientific \& Engineering Research, 8(7), 653-660.
Rohloff, J. (2015). Analysis of phenolic and cyclic compounds in plants using derivatization techniques in combination with GCMS-based metabolite profiling. Molecules, 20(2), 3431-3462.

Suja, D., Bupesh, G., Rajendiran, N., Mohan, V., Ramasamy, P., Muthiah, N. S., Arul, A. E., Meenakumari, K., \& Prabu, K. (2017). Phytochemical screening, antioxidant, antibacterial activities of Citrus limon and Citrus sinensis peel extracts. International Journal of Pharmacognosy and Chinese Medicine, 1(2): 000108.

Tamil, S. S., Jamuna, S., Thekan, S., \& Paulsamy, S. (2017). Profiling of bioactive chemical entities in Barleriabuxifolial using GC-MS analysis-a significant ethno medicinal plant. Journal of Ayurvedic and Herbal Medicine, 3(2), 63-77

Uraku, A. J., \& Igwenyi, I. O. (2016). Comparative studies of phytochemical and vitamin constituents of Citrus sinensis and Vitisvinitera peels. EC Nutrition, 4(2), 812-816.

Uraku, A. J., Uraku, H. O., Nweke F. N., Enemecukwu, B. N. (In press). GC-MS Technique Determination of Phytocomponents in Emilia pretermissa Milne-Redh leaves. Kuwait Medical Journal.

Uraku, A. J., Uraku, O. H., Nweke, F. N., Orji, O. U., Igwenyi, I. O., Edwin, N., Ezeani, N. N. (2018). Phytochemical and GCMS evaluation of bioactive principle of Vitis vinifera peels. Asian Journal of Applied Sciences, 11(4),192-198. 